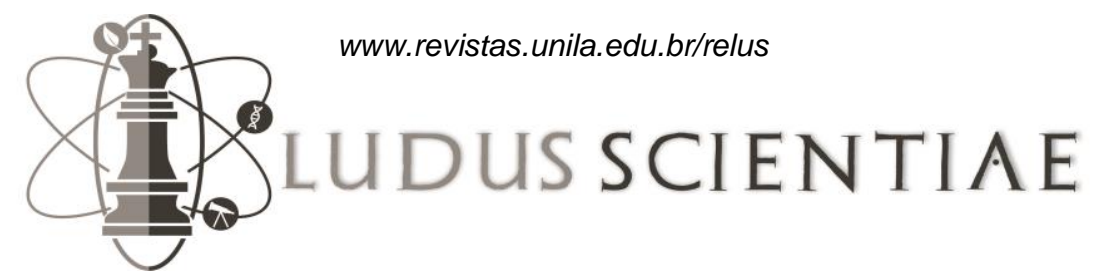

Revista eletrônica Ludus Scientiae - (RELuS) | V. 2, N. 1, Jan./Jun., 2018.

\section{JUEGOS Y GAMIFICACIÓN EN LAS CLASES DE CIENCIA: UNA OPORTUNIDAD PARA HACER MEJOR CLASE O PARA HACER MEJOR CIENCIA?} Games and gamification in science classes: an opportunity to improve the "doing of lessors" or to
improve the "doing of science"?

Jogos e gamificação em aulas de ciências: uma oportunidade de fazer melhor aula ou fazer melhor ciência?

\section{Resumo}

Enquanto o jogo tem sido uma ferramenta de ensino e aprendizagem muito comum, nos últimos anos, o surgimento de videogames digitais e das estratégias de gamificação colocou, de volta à cena, essa aproximação educacional. Com este artigo, queremos propor uma vista sobre o jogo na sala de aula das ciências experimentais, conforme se é usado para implicar os alunos/ estudantes na prática tradicional de uma aula (o que chamamos "fazer aula") ou na prática científica (o que chamamos de " fazer ciência "). Para fazer isso, analisaremos e compararemos dois jogos de mesa, dois videogames e duas estratégias de gamificação direcionada no ensino e aprendizagem nos diferentes temários de química e biologia.

Palavras-Chave: videogame, gamification, práctica científica.

\section{Abstract}

Despite gaming has always been a very common instructional approach, in recent years the emergence of digital videogames and gamification strategies have staged it again. In this article we propose a perspective on science educational games, differentiating whether they are used to involve students in traditional classroom practices (what we call "doing lessons") or in scientific practices (what we call "doing science"). To do so, we analyze and compare two board games, two videogames and two gamification scenarios, all of them devoted to chemistry and biology education. Keywords: gamification, videogames, scientific practices.

\section{Resumen.}

Si bien el juego ha sido siempre una herramienta de enseñanza y aprendizaje muy común, en los últimos años la eclosión de los videojuegos digitales y las estrategias de gamificación ha puesto de nuevo en escena esta aproximación educativa. Con este artículo queremos proponer una mirada al juego en el aula de ciencias experimentales, según si se usa para involucrar a los estudiantes en la práctica tradicional de aula (lo que denominamos "hacer clase") o en la práctica científica (lo que denominamos "hacer ciencia"). Para ello, analizamos y comparamos dos juegos de mesa, dos videojuegos y dos estrategias de gamificación enfocadas a la enseñanza y aprendizaje de diferentes contenidos de química y biología.

Palabras clave: gamificación, videojuegos, práctica científica.

\section{AUTORES:}

VÍCTOR LÓPEZ ${ }^{1}$

ORCID 0000-0002-2161-9211

${ }^{1}$ Instituto de Secundaria

Pau Vila

JORDI DOMÈNECH-CASAL ${ }^{2}$

ORCID 0000-0002-7324-0000

${ }^{2}$ Instituto de Secundaria Marta Estrada

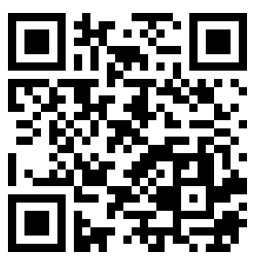

Para citar este artigo:

LÒPEZ, V.; DOMÈNECHCASAL, J. Juegos $y$ gamificación en las clases de ciencia: una oportunidad para hacer mejor clase o para hacer mejor ciencia?. Revista Eletrônica Ludus Scientiae, Foz do Iguaçu, v. 02, n. 01, p. 34-44, jan./jun., 2018. 


\section{INTRODUCCIÓN}

El desarrollo de la competencia científica implica, más allá de elementos basales como el léxico y la enunciación de los modelos científicos (ciclo del agua, estructura del átomo...) o la aplicación de algoritmos (fórmulas de dinámica, ejercicios de genética...), elementos complejos como el desarrollo de habilidades de razonamiento y la comprensión de los marcos epistémicos del conocimiento científico. Las metodologías transmisivas específicas tradicionales fracasan en estos objetivos y desde hace tiempo se viene reclamando una enseñanza de las ciencias más activa, problematizada y contextualizada. Fruto de ello son el auge de las metodologías de enseñanza de las ciencias mediante la indagación o modelización (CAAMAÑO, 2011; COUSO, 2014) o el aprendizaje basado en proyectos (SANMARTÍ y MÁRQUEZ, 2017).

En este marco, que persigue también encontrar las fórmulas didácticas para cumplir con la demanda pedagógica de situar al alumno en el centro del proceso de aprendizaje, ha emergido con fuerza una variedad de tendencias educativas que se basan en el uso del juego y de la gamificación como estrategias de enseñanza y aprendizaje. Con este artículo queremos discutir cómo el juego y la gamificación encajan con una enseñanza de las ciencias que involucre a los estudiantes a hacer ciencia, proponiendo tentativamente una taxonomía según si se busca "hacer mejor clase" o "hacer mejor ciencia”.

\section{Los juegos educativos y la gamificación}

El juego es un sistema de reglas que definen un conflicto artificial, donde la participación de los jugadores en este conflicto se traduce en un resultado cuantificable (SALEN y ZIMMERMAN, 2004). El juego (y la actividad de jugar que lleva asociada) siempre ha sido, de hecho, un elemento central en el desarrollo físico, cognitivo y social de las personas, especialmente de aquellas de edades tempranas (niños y jóvenes), y por lo tanto ha estado intrínsecamente relacionado con los procesos de enseñanza y aprendizaje que se dan a lo largo de la escolaridad (BISHOP et al., 2008) y ha centrado la atención de múltiples tendencias educativas que han puesto desde hace décadas la idea del juego como centro de la actividad de los estudiantes (YAGER, 1991). En todo el mundo existe una extensa tradición de emplear juegos como elemento didáctico, que según el contexto educativo y la finalidad de su uso pueden ser individuales o colectivos, tradicionales o adaptados, dirigidos o libres, para adultos, jóvenes o niños, de piezas o de tablero, de pensamiento lógico o de acción, de azar o de estrategia, etc. (YVERN, 1998).

A pesar de esta extensa tradición, en los últimos años la presencia del juego como elemento didáctico ha tomado especial relevancia en la educación formal, principalmente debido a dos factores. Por un lado, la progresiva digitalización de las aulas en forma de dispositivos electrónicos (tabletas, ordenadores, pizarras digitales, etc.) ha llevado a la eclosión de los videojuegos educativos (en algunos casos, llamados serious games) (ULISAK, 2010). Por otro lado, la aparición en los últimos años de la idea de gamificación plantea el uso de los elementos del juego en contextos no lúdicos (DETERDING, 2011). Si bien este término no proviene originalmente del ámbito educativo, sí que ha tenido una importante implantación en las aulas gracias a la gran cantidad de recursos, plataformas y entornos virtuales para trabajar en el aula mediante gamificación. Actualmente una gran cantidad de editoriales, escuelas, grupos de innovación educativa, etc., ofrecen cursos, talleres y actividades para que el profesorado conozca qué recursos en forma de juego tiene a su disposición, qué maneras tiene de introducir elementos de juego en sus clases e incluso cómo puede "gamificar" sus clases tradicionales. Para ello, diferentes autores han establecido una estructura común en todo contexto gamificado (WERBACH y HUNTER, 2012; CORNELLÀ, 2015; RIPOLL, 2016), proponiendo que la gamificación requiere de unas dinámicas de juego (las limitaciones, la narrativa que se presenta y las emociones que evoca, el sentido de progresión y de mejora a lo largo del juego, las relaciones que se establecen entre jugadores, etc.), unas mecánicas (los retos que se plantean, los turnos en que se estructura el juego, las recompensas, la manera en que se coopera o compite, etc.), y finalmente los componentes 
específicos del juego (los puntos o recompensas que se consiguen en el juego, los avatares de los jugadores, los niveles a superar, etc.).

Este importante movimiento que aboga por el juego y la gamificación como estrategia educativa se basa en gran medida en la idea que la participación en un juego implica una reacción emocional del jugador, provocada por el propio desafío del juego, el feedback y la interacción constante que se produce en el proceso de jugar y el resultado cuantificable que se deriva del hecho de jugar (ganar, perder, pasar al siguiente nivel, etc.). Esta idea, de hecho, está estrechamente ligada a la teoría de la motivación intrínseca y extrínseca, muy usada en el campo de la psicología educativa, que propone distinguir aquellas demandas que no requieren de incentivos ni castigos porque son intrínsecamente gratificantes en sí mismas de aquellas que para ser realizadas requieren la existencia de un rédito extrínseco a la demanda (WOOLFOLK, 2006). Desde este marco, diferentes investigaciones han identificado efectos positivos del juego y la gamificación en numerosos aspectos emocionales y actitudinales de loes estudiantes. Torres (2002) destaca el papel del juego como herramienta que puede estimular la concentración, la reflexión, en desarrollo de estrategias e incluso aspectos del ámbito ético (honradez, solidaridad) y emocional (dominio de sí mismo, gestión de la frustración, ...). Kapp (2012) plantea que el uso de las mecánicas de juego, de estéticas de juego y de pensamiento de juego ('game thinking' en inglés) permiten involucrar a las personas, motivar su acción y promover su aprendizaje y su resolución de problemas. Emmer y Stough, (2010) señalan la incidencia de estrategias de este tipo en la gestión de aula y en la organización de las actividades, así como para captar mejor la atención del alumnado y favorecer su cooperación. No obstante, esta incipiente bibliografía también indica que la eficacia de la gamificación no es per se, sino que depende de varias componentes, y en particular de los participantes y el contexto en que se desarrolla la gamificación (HAMARI, KOIVISTO y SARSA, 2014) y algunos estudios empiezan a distinguir tipos y enfoques sobre gamificación que permiten diferenciar estos efectos en los distintos tipos de gamificación (CAPONETTO, EARP y OTT, 2014; DICHEVA et al., 2015).

\section{Más allá de motivar: Involucrar a los estudiantes en las prácticas científicas}

Para Morris et al. (2013), estos "andamios motivacionales" (los aspectos competitivos del juego, los niveles de progreso, los escenarios cercanos a los intereses del alumnado, los premios, etc.,) no bastan, sino que se requiere la existencia de andamios cognitivos (simulaciones, habilidades de razonamiento, etc.) y de dinámicas metacognitivas en el juego. Además, el exceso de énfasis en este nivel motivacional tan común en el mainstream gamificador parece sugerir que todo se basa en la necesidad de añadir elementos de recompensa (o motivación extrínseca) a la experiencia del aprendizaje, que por sí sola no es suficiente motivante. A nuestro entender, si bien parecen evidentes las aportaciones de este enfoque a corto plazo al desarrollo de los elementos basales de la competencia científica mencionados al inicio de este artículo (mediante juegos memorísticos o la iteración de prácticas académicas), no queda claro qué papel puede jugar este enfoque en el desarrollo de estos elementos a largo plazo o de los elementos complejos (razonamiento y epistemología).

Esta centralidad de los elementos basales en el aprendizaje que a menudo promueve la gamificación contrasta con la apuesta cada vez más decidida en el campo de la didáctica de las ciencias por una enseñanza y aprendizaje de ideas científicas que no se base en la transmisión y reproducción de contenidos científicos tradicionales, sino en la participación de la práctica social, discursiva y cognitiva que lleva asociada la actividad científica (IZQUIERDO-AYMERICH, SANMARTÍ y ESPINET, 1999; OSBORNE, 2014). El marco de la práctica científica definido en los K-12 Next Generation Science Standards (NRC, 2012) concreta ocho prácticas científicas clave que sintetizan el "hacer ciencia", y por lo tanto, sobre las que debería orbitar la actividad científica en el aula: plantearse preguntas científicamente relevantes e investigables, desarrollar y usar modelos científicos, planificar y llevar a cabo investigaciones, analizar e interpretar datos experimentales, usar pensamiento computacional y matemático, construir explicaciones y diseñar soluciones, argumentar científicamente en base a pruebas y comunicar a la comunidad los resultados de la actividad científica (BYBEE, 2011). Del mismo 
modo, Osborne (2014) propone considerar tres esferas de la práctica científica en el aula: la modelización (desarrollar, evaluar y refinar explicaciones científicas sobre fenómenos naturales), la indagación (diseñar y llevar a cabo experimentos y analizar e interpretar datos) y la argumentación (comunicar las ideas propias y las interpretaciones de los resultados para persuadir a los demás de su validez).

Si bien en algunos casos las actividades gamificadas incorporan escenarios o roles de interés epistemológico o prácticas científicas, esto no siempre es así, y en algunos casos su uso no parece orientado a hacer al alumno partícipe de una experiencia científica, sino hacer la clase más "participativa" con la excusa de un escenario científico, obviando sus elementos clave. Así pues, del mismo modo que Barab y Dede (2007) destacan la función de algunas simulaciones "para hacer (y no solo para recibir ciencia)", nos preguntamos qué criterios permiten decidir sobre la idoneidad de usar un juego, un videojuego o una estrategia de gamificación en el aula, basándonos en qué concepciones de enseñanza y aprendizaje subyacen en su diseño didáctico.

\section{UNA PROPUESTA DE TAXONOMIA ENTRE JUEGOS PARA “HACER MEJOR CLASE” O PARA "HACER MEJOR CIENCIA"}

Con el objetivo final de compartir criterios didácticos para la selección de juegos como herramienta educativa en las clases de ciencia, nos proponemos analizar y comparar las oportunidades que ofrecen algunos juegos y estrategias de gamificación. No queremos simplemente promover que los estudiantes estén más motivados en clase de ciencia a través del juego, sino que se involucren activamente en actividades de carácter científico (prácticas científicas) descritas anteriormente. Para ello, proponemos una taxonomía que distingue entre si los sistemas de reglas que configuran el juego se conciben como un artefacto didáctico para "hacer mejor clase" o bien para "hacer mejor ciencia", y que se organiza según lo que en la teoría de gamificación se denominan los elementos estructurales del juego: las dinámicas y las mecánicas. Esto no significa que existan únicamente dos tipos de juego educativo ni que todos los juegos educativos de ciencias puedan organizarse en dos bloques monolíticos, sino que, en una rica variedad de juegos con múltiples matices, según en qué aspectos pedagógicos o didácticos ponga énfasis el juego, pueden observarse dos tipos de tendencias o aproximaciones.

Cuadro 1: Taxonomía del juego según si promueve "hacer clase" o "hacer ciencia".

\begin{tabular}{|c|c|c|}
\hline $\begin{array}{l}\text { Elemento } \\
\text { estructural del } \\
\text { juego }\end{array}$ & Juego para "hacer mejor clase" & Juego para "hacer mejor ciencia" \\
\hline $\begin{array}{l}\text { Dinámicas: } \\
\text { ¿Qué dinámicas se } \\
\text { reproducen y qué tipo } \\
\text { de motivación hay } \\
\text { detrás? }\end{array}$ & $\begin{array}{l}\text { Las dinámicas del juego reproducen } \\
\text { las que se dan a menudo en una clase } \\
\text { tradicional, donde existe un } \\
\text { conocimiento académico } \\
\text { establecido (ya sea conceptual o } \\
\text { procedimental, pero a menudo de } \\
\text { carácter memorístico y reproductivo), } \\
\text { o bien unas normas de actitud. Más } \\
\text { allá del grado de competición, lo que } \\
\text { mueve al estudiante a asimilar este } \\
\text { conocimiento es la recompensa que } \\
\text { hay durante el juego o al final de él, } \\
\text { vinculada a la calificación académica } \\
\text { del estudiante (mejorar la nota en la } \\
\text { evaluación). Se trata, por lo tanto, de } \\
\text { una motivación extrínseca. }\end{array}$ & $\begin{array}{l}\text { Las dinámicas del juego reproducen las que se } \\
\text { dan en la actividad profesional de las ciencias, } \\
\text { donde existe un reto intelectual (ya sea } \\
\text { construir una explicación, diseñar una } \\
\text { solución a un problema, disfrutar de un placer } \\
\text { estético, etc.), sin cuya superación no se } \\
\text { puede progresar en el juego. Más allá del } \\
\text { grado de competición, lo que mueve al } \\
\text { estudiante a enfrentarse a este reto está } \\
\text { vinculado con la necesidad de dar sentido y } \\
\text { orden a las cosas, a la curiosidad, a la } \\
\text { autosuperación, etc. Se trata, por lo tanto, de } \\
\text { una motivación intrínseca. }\end{array}$ \\
\hline $\begin{array}{l}\text { Mecánicas: } \\
\text { ¿Qué mecánicas } \\
\text { conducen la acción y }\end{array}$ & $\begin{array}{l}\text { El juego gira entorno a los retos que se } \\
\text { le plantea al estudiante de demostrar } \\
\text { hasta qué punto sabe o domina un }\end{array}$ & $\begin{array}{l}\text { El juego gira en torno al desarrollo de } \\
\text { conocimientos, competencias, habilidades, } \\
\text { actitudes, etc., de forma instrumental a lo }\end{array}$ \\
\hline
\end{tabular}


cómo se relaciona el conocimiento académico, mantiene estudiante con el una actitud o interés académico, conocimiento a posee unas habilidades académicas... través de ellas? $\quad y$, por lo tanto, que se mide también con acciones de carácter académico (responder preguntas, resolver ejercicios, cumplir normas, etc.) más o menos vestidas de juego. Además, los sistemas de puntos y recompensas específicos que conducen la acción suelen reproducir la relación académica profesor-alumno: el profesor "otorga" los puntos de forma directa o indirecta, y estos tienen una correspondencia con la nota, es la autoridad académica quien dirige la evaluación sumativa. largo del juego, por lo que progresar implique saber (o saber hacer) algo que antes de empezar a jugar no se sabía (o no se sabía hacer), y por lo tanto el progreso del juego promueve la autorregulación del aprendizaje. Además, el juego puede poner el énfasis en el componente epistémico (cómo se está construyendo el conocimiento), haciendo que el sistema de puntos y recompensas que conducen la acción reproduzcan las relaciones sociales propias de la actividad científica (validación del conocimiento, consenso social, recompensa en forma de capacidad para seguir haciendo ciencia, etc).

Fuente: Elaborado por los autores.

A continuación discutimos esta propuesta taxonómica analizando y comparando seis escenarios donde hay juegos que pertenecen a tres formatos lúdicos distintos: (1) el juego de mesa, (2) el videojuego digital y (3) la aventura gamificada. Para cada uno de estos 3 formatos lúdicos, se describen dos escenarios comparándolos entre ellos. Si bien estos escenarios son cercanos a la experiencia profesional de los autores y adaptados para la ocasión, el objetivo no es documentar en detalle ninguna experiencia educativa concreta llevada en un contexto determinado, sino ejemplificar de la forma más paradigmática posible sobre nuestra propuesta de taxonomía. Para ello, también se ha procurado seleccionar tres temáticas científicas distintas que comprenden distintos contenidos curriculares de química y biología, para poder así comparar juegos entre ellos más fácilmente.

\section{JUEGOS DE MESA Sobre estructura atómica}

Existen muchos ejemplos de juegos de mesa relacionados con contenidos científicos. Juegos de cartas con preguntas-respuesta, otros con tableros y fichas, de piezas y construcción, de rol, entorno a la resolución de enigmas, etc. En este caso nos centramos en dos juegos con un tablero, pero con dos usos muy distintos.

Cuadro 2: Escenarios "Juego de la oca de la tabla periódica" y "Juego de las formas misteriosas".

\begin{tabular}{|c|c|}
\hline Juego para "hacer mejor clase" & Juego para "hacer mejor ciencia" \\
\hline $\begin{array}{l}\text { El juego de la oca de la tabla periódica consiste en } \\
\text { hacer avanzar unas piezas de juego sobre un } \\
\text { tablero con un recorrido de casillas, donde cada } \\
\text { casilla corresponde a un elemento de la tabla } \\
\text { periódica (ver Figura } 1 \text { - izquierda). Mediante } \\
\text { dados, los jugadores (ya sea individualmente o en } \\
\text { grupos) van avanzando casillas y deben responder } \\
\text { a alguna cuestión relacionada con la configuración } \\
\text { atómica del elemento (su número atómico, } \\
\text { másico, sus electrones de valencia, su } \\
\text { nomenclatura, etc.). } \\
\text { Si saben la respuesta pueden seguir avanzando en } \\
\text { el siguiente turno. Si no saben la respuesta, deben } \\
\text { retroceder, de manera que se evalúa el } \\
\text { conocimiento sobre la tabla periódica que tienen } \\
\text { los jugadores. Gana quien antes llegue a la meta. }\end{array}$ & $\begin{array}{l}\text { El juego de las formas misteriosas consiste en adivinar la } \\
\text { forma de una pieza de madera puesta debajo de un } \\
\text { tablero que la oculta (ver Figura } 1 \text { - derecha). Los } \\
\text { jugadores deben lanzar canicas que crucen el tablero por } \\
\text { debajo, y analizar su trayectoria visible para deducir por } \\
\text { donde ha podido rebotar y por lo tanto cual puede ser la } \\
\text { forma de la pieza de madera que se oculta (analogía } \\
\text { mecánica del experimento de Scattering de Rutherford } \\
\text { donde puso a prueba el modelo Atómico de Thomson, } \\
\text { que puede hacerse explícita con los estudiantes para } \\
\text { aportar una visión epistémica). Con cada lanzamiento, } \\
\text { obtienen nueva información que deben usar para poner a } \\
\text { prueba su modelo (la forma que creen que tiene su pieza } \\
\text { de madera) e ir sofisticándolo. Gana el grupo que describa } \\
\text { con mayor detalle la forma con el menor número de } \\
\text { lanzamientos. }\end{array}$ \\
\hline
\end{tabular}

Fuente: Elaborado por los autores. 



Figura 1: Izquierda: Tablero del juego de la oca de la tabla periódica (Fuente: Blog "Lápiz, papel, acción": http://lpadigital.blogspot.com/ ). Derecha: Juego de las formas misteriosas. En la parte superior derecha, el aspecto del tablero ocultando la figura. En la parte inferior derecha, la forma de la pieza de madera una vez destapada (Fuente: Elaborada por los autores).

\section{Videojuegos digitales sobre redes tróficas}

Igual como ocurre con los juegos de mesa, también existen muchos ejemplos de videojuego para ser usado en la enseñanza de las ciencias: tipo arcade, tipo plataforma, tipo sandboxes, tipo quizz, así como de puntería, de estrategia, de simulación, de conducción, de construcción, etc. En este caso presentamos dos escenarios con dos juegos online gratuitos, ambos relacionados con la nutrición y las redes tróficas.

Cuadro 3: Escenarios "juego de la oca de la tabla periódica" y "juego de las formas misteriosas".

\begin{tabular}{|c|c|}
\hline Juego para "hacer mejor clase" & Juego para "hacer mejor ciencia" \\
\hline $\begin{array}{l}\text { En el Quizz "Quién se come a quien" el } \\
\text { estudiante se enfrenta a preguntas de “¿Quién se } \\
\text { come a...?”, ante las que debe seleccionar entre } \\
\text { tres opciones que le da la interfaz del juego (Figura } \\
\text { 2- izquierda). En un tiempo límite, el estudiante } \\
\text { debe responder cual es la relación depredador- } \\
\text { presa correcta, para así acumular el máximo de } \\
\text { puntos. Si acierta la respuesta, se le suma un punto } \\
\text { al marcador y se pasará a la siguiente pregunta. Si } \\
\text { se falla, la opción seleccionada se muestra de color } \\
\text { rojo y se debe elegir otra. }\end{array}$ & $\begin{array}{l}\text { En el juego "Food Fight" cada jugador ( } 2 \text { en total) elige } \\
\text { una especie animal, y gana el jugador que al final del } \\
\text { juego consigue tener más individuos de su especie vivos. } \\
\text { El juego en todo momento ofrece información sobre } \\
\text { quién se come a quién, a partir de la cual cada jugador } \\
\text { debe tomar decisiones sobre cómo aumentar el número } \\
\text { de individuos de su especie y a la vez reducir el número } \\
\text { de individuos de la especie rival (Figura } 2 \text { - derecha), } \\
\text { ideando estrategias basadas en el modelo de red trófica } \\
\text { (añadiendo presas, depredadores, competidores, etc.), } \\
\text { que debe usar para predecir la dinámica de población de } \\
\text { cada especie y poder así tomar decisiones en el juego que } \\
\text { le beneficien para ganar. }\end{array}$ \\
\hline
\end{tabular}

Fuente: Elaborado por los autores. 

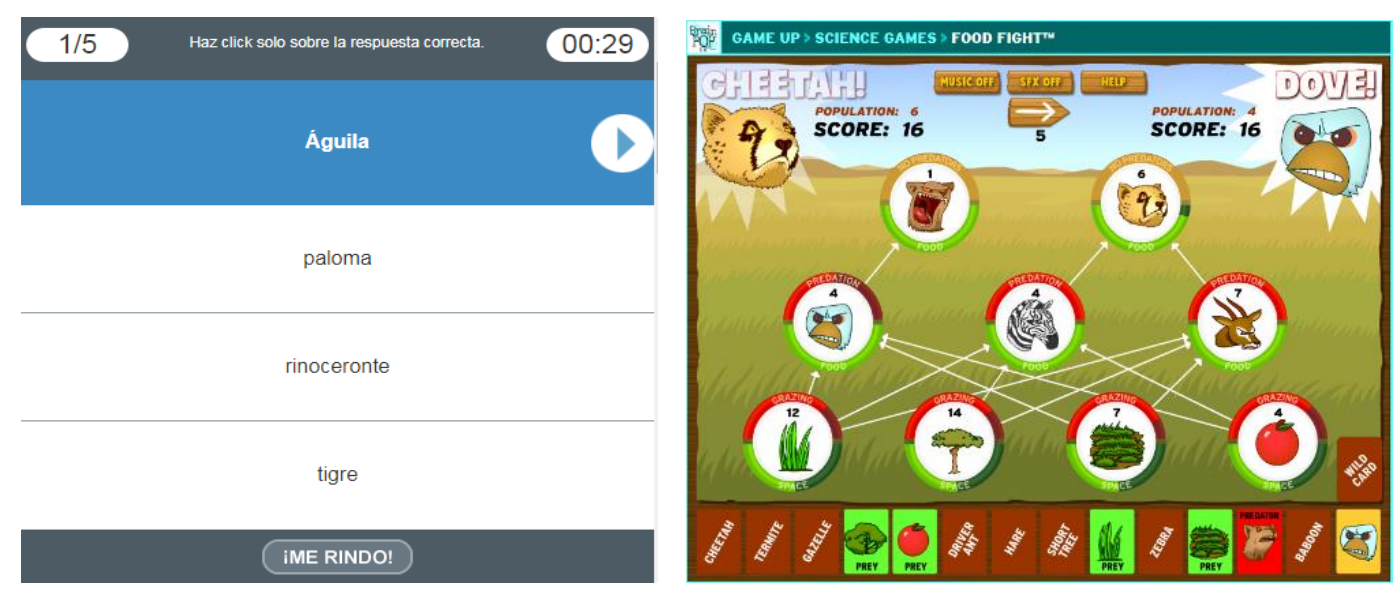

Figura 2: Izquierda: Juego “¿Quién se come a quién?” (Fuente: Obtenida del web: https://www.cerebriti.com/juegos-de-ciencias/-quien-come-a-quien\#.W2A_lNUzZhE ). Derecha: Juego "Food Fight" (Obtenida del web: https://www.brainpop.com/games/foodfight/).

\section{AVENTURAS GAMIFICADAS SOBRE LA EVOLUCIÓN}

En las aventuras gamificadas se incorpora como elemento didáctico un itinerario hacia un objetivo final en el que los alumnos desarrollan algún tipo de rol. Suelen incorporar personajes, avatares, puntos, ranquings, badges, y existen una gran diversidad de entornos, si bien quizás el más conocido sea ClassDojo ${ }^{1}$. También en este tipo de gamificación se plasman los dos enfoques para "hacer mejor clase" o "hacer mejor ciencia" (Cuadro 3).

Cuadro 3 - Escenarios "Juego de la oca de la tabla periódica" y "Juego de las formas misteriosas".

\begin{tabular}{|c|c|}
\hline & \\
\hline $\begin{array}{l}\text { la Gamifica } \\
\text { anás" difere } \\
\text { errizado en d } \\
\text { a, con el ob } \\
\text { rá posible si }\end{array}$ & $\begin{array}{l}\text { En la Gamificación "La is } \\
\text { Caminalcules" diferentes gru } \\
\text { aterrizado en diferentes punt } \\
\text { con el objetivo de descubrir cór } \\
\text { Para investigar la isla, } \\
\text { progresivamente muestras de } \\
\text { - derecha), y tienen que } \\
\text { filogenético con la infor } \\
\text { Periódicamente presentan sus } \\
\text { científico ( } 1 \text { vez al mes), y cada } \\
\text { que usará para evaluar las pres } \\
\text { demás grupos. Disponen de 1o } \\
\text { entre los demás grupos segú } \\
\text { presentado. Según los puntos c }\end{array}$ \\
\hline
\end{tabular}

Fuente: Elaborado por los autores. 


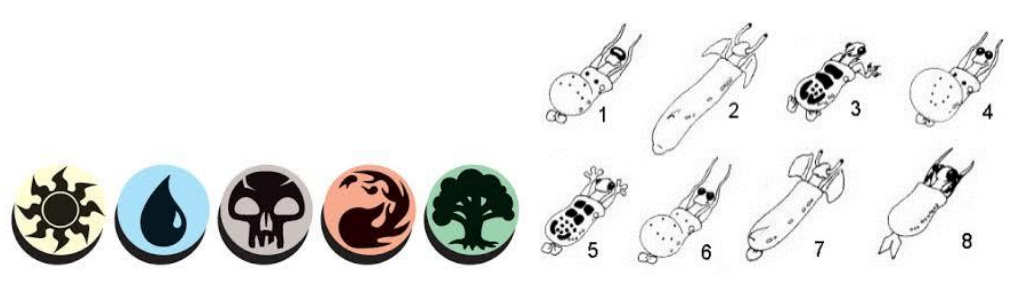

Figura 3 : Izquierda: Imágenes de los manás para usar en "La isla misteriosa de los manás” (Fuente: Obtenida de la web oficial: http://gatherer.wizards.com). Derecha: Dibujos originales de los Caminalcules para usar en "La isla misteriosa de los caminalcules" (Fuente: Obtenida del artículo de McComas y Alters (1994)).

\section{Dinámicas y propósito en la gamificación}

Si bien la motivación ha sido propuesta como uno de los beneficios de la gamificación, consideramos que las dinámicas de los ejemplos propuestos ofrecen distintas visiones de ello, en relación al propósito que se comunica al alumnado. En algunas actividades, el propósito que se comunica al alumnado (ganar una competición, tener mejor nota) implica vincular al alumnado a la actividad mediante una motivación extrínseca, reforzando un vínculo retributivo -tú aprendes, yo te pago- extraño al aprendizaje. En otras, en cambio, la motivación se promueve mediante un reto asumible, un problema o enigma a resolver que da sentido (no sólo propósito) por sí mismo a la actividad, lo que identificamos como motivación intrínseca.

Aunque compartimos la necesidad de buscar formas más activas y motivadoras para la enseñanza, lo cierto es que el uso de motivaciones extrínsecas debe considerarse con precaución, por distintas razones: 1) Ubicar el aspecto motivador de una gamificación en una parte de la actividad alejada de los contenidos comunica al alumnado la idea errónea que los contenidos no pueden, por si mismos, ser motivadores en determinados contextos. 2) el uso de insignias o badges, intercambios de puntos y similares implica una dinámica evaluadora pobre: el feedback obtenido es limitado y sólo indica aciertos y errores, sin incluir acompañamiento y promueve dinámicas consumistas y aceleradas poco compatibles con el ritmo pausado y la reflexión y argumentación que requieren algunos conceptos o procedimientos científicos; 3) Incluso considerando compaginar ambas motivaciones (intrínseca y extrínseca) muchos estudios demuestran que ofrecer recompensa externa para un comportamiento internamente gratificante puede conducir a una reducción de la motivación intrínseca, un fenómeno conocido como efecto de sobrejustificación.

El hecho que los juegos incluyan dinámicas competitivas no es un problema necesariamente (existen también en el mundo del conocimiento científico), pero debe valorarse desde un punto de vista epistemológico la oportunidad de que estas dinámicas en algún momento redunden en eventos vinculados a la idea de "construir juntos", epistemológicamente más coherente.

\section{Mecánicas y narración en la gamificación}

En lo que respecta a las mecánicas, hemos descrito dos relaciones distintas con la narración del juego. Por un lado, juegos que reproducen mecánicas propias de aula, mencionando incluso explícitamente elementos que le son propios (tests, capacidad de usar chuletas en examen). En estos juegos (juego de la oca, quizz y de los manás) la narración de la acción no tiene relación alguna con los contenidos, que son accesorios: de hecho, podrían usarse -sin cambiar las mecánicas- los mismos juegos para otros contenidos distintos. La profundidad de desarrollo de los contenidos no se beneficia del juego, puesto que la metodología de aprendizaje se mantiene como metodología transmisiva basada en la memorización y reproducción. 
En cambio, en los otros juegos propuestos la narración crea contextos que emulan procesos de razonamiento y epistémicos propios de las ciencias (modelizar en las Formas misteriosas, hacer predicciones en Food Fight, construir conocimiento y conseguir financiación para la investigación en la Isla de los Caminalcules) en los que los conceptos que se quieren desarrollar tienen un papel instrumental, central en el juego. Desde un punto de vista del aprendizaje es evidente que los conceptos se adquieren mejor en su instrumentalización en el marco de una narración o contexto coherente que en su reproducción descontextualizada. De hecho, esta es la base de propuestas pedagógicas como el Aprendizaje Basado en Proyectos (KILPATRICK, 1918) y su concreción en la resolución de problemas o Estudios de Caso (LARMER, MERGENDOLLER y BOSS, 2015, SANMARTÍ, 2016, DOMĖNECH-CASAL, en edición) metodologías que podemos considerar cercanas a los ejemplos de juegos "Para hacer ciencia" propuestos.

Teniendo en cuenta lo propuesto para las dinámicas y mecánicas, podemos concluir que cuanto más lejana esté la narración y el propósito del juego de los contenidos a trabajar, menor será la utilidad del juego para aprender ciencias a largo plazo. Por dos razones: 1) por un lado, una narración lejana (p.e.: Isla de los manás) promueve que el alumnado se implique en la actividad, no en el campo de conocimiento, lo que a largo plazo podemos prever que resultará en alumnos más vinculados a la actividad escolar que a la ciencia. 2) por otro lado, una narración lejana no permite la instrumentalización de los contenidos ni la presencia de mecánicas promotoras de razonamientos o eventos epistémicos (que permitirían un mayor nivel de apropiación de los contenidos y del discurso de la ciencia).

\section{La gamificación, en la encrucijada}

La gamificación está experimentando un rápido auge que requiere propuestas que faciliten el análisis de su utilidad en distintos aspectos (motivación, profundidad y duración de los aprendizajes, utilidad para transmitir actitudes y valores, ...). La taxonomía que proponemos establece una separación en dos ámbitos según el propósito educativo: Para hacer clase o para hacer ciencia, que difieren en el papel de las dinámicas y las mecánicas.

Otras aproximaciones al juego que no hemos contemplado son las actividades de juego que no tienen ningún propósito explícito, como el Tinkering (SIMARRO y COUSO, 2016) o las plataformas o elementos que -habiendo sido originalmente concebidos para el juego (como Minecraft o Lego)- se usan para actividades didácticas como la construcción o recreación de escenarios geológicos o históricos que no constituyen propiamente una gamificación (puesto que no atiende a dinámica ni mecánicas gamificadoras).

Existe actualmente entre el profesorado un gran interés por las nuevas metodologías y la gamificación está siendo objeto de implantación en las aulas. Los ejemplos que hemos propuesto pretenden ilustrar que no toda gamificación implica innovación educativa, y que algunas modalidades de gamificación pueden incluso reforzar metodologías tradicionales o transmisivas, presentándolas como pedagógicamente innovadoras. En la aplicación en el aula, nuestra propuesta de taxonomía puede ser de ayuda al profesorado para diferenciar e identificar actividades que supongan una incidencia real en la enseñanza de la ciencia, más allá de la motivación del alumnado. En concreto, las tablas presentadas relativas a las diferencias en lo relativo a las mecánicas y dinámicas serán una ayuda para el profesorado interesado en seleccionar actividades basadas en el juego más relevantes para sus clases. El potencial del juego y gamificación para involucrar a los estudiantes en prácticas científicas es inmenso. ¡Aprovechémoslo!

\section{REFERENCIAS}

BARAB, D.; DEDE, C. Games and Immersive Participatory Simulations for Science Education: An Emerging Type of Curricula. Journal of Science Education and Technology, v. 16, n. 1, p. 1-3, 2007. 
BISHOP, A. J. El papel de los juegos en educación matemática. En: Bañeres, D. et al. El juego como estrategia didáctica. Barcelona: Graó, p. 23-33, 2008.

CAAMAÑO, A. Contextualización, indagación y modelización. Tres enfoques para el aprendizaje de la competencia científica en las clases de química. Aula de Innovación Educativa, n. 207, p. 17-21, 2011.

CAPONETTO, I.; EARP, J.; OTT, M. Gamification and Education: A Literature Review. ECGBL2014-8th, European Conference on Games Based Learning, 2014, p. 50.

COUSO, D. De la moda de "aprender indagando" a la indagación para modelizar: una reflexión crítica. XXVI Encuentro de Didáctica de las Ciencias Experimentales. Huelva (Andalucía), 2014.

DETERDING, S. et al. Gamification: Toward a Definition. CHI 2011. Vancouver: CHI, 2011.

DICHEVA, D.; DICHEV, C.; AGRE, G.; ANGELOVA, G. Gamification in Education: A Systematic Mapping Study. Educational Technology \& Society, v. 18, n. 3, p. 75-88, 2015.

DOMÈNECH-CASAL, J. (en edición). Contexto y modelo en el Aprendizaje Basado en Proyectos. Apuntes para el ámbito científico. Alambique, Didáctica de las Ciencias Experimentales, (En edición).

EMMER, E. T.; STOUGH, L. M. Classroom Management: A Critical Part of Educational Psychology, With Implications for Teacher Education. Educational Psychologist, v. 36, n. 2, p. 103-112, 2001.

HAMARI, J.; KOIVISTO, J.; SARSA, H. Does Gamification Work? - A Literature Review of Empirical Studies on Gamification. 47th Hawaii International Conference on System Science, p. 3025-3034, 2014.

IZQUIERDO-AYMERICH, M.; SANMARTÍ, N.; ESPINET, M. Fundamentación y diseño de las prácticas escolares de ciencias experimentales. Enseñanza de las Ciencias, v. 17, n. 1, p. 45-59, 1999.

KAPP, K. M. The Gamification of Learning and Instruction: Game-based Methods and Strategies for Training and Education. San Francisco: John Wiley \& Sons, 2012, p. 1-18.

KILPATRICK, W. E. The Project Method: the use of the purposeful act in the educative process. New York: Teachers college, Columbia University, p. 6-12, 1918.

LARMER, J.; MERGENDOLLER, J.; BOSS, S. Setting the Standard for Project Based Learning: A Proven Approach to Rigorous Classroom Instruction. Alexandria: ASCD, p. 5-12, 2015.

MCCOMAS, W. F.; ALTERS, B. J. Modeling modes of evolution: Comparing phyletic gradualism and punctuated equilibrium. The American Biology Teacher, v. 56, n. 6, p. 354-36o, 1994.

MORRIS, B. J.; CROKER, S.; ZIMMERMAN, C.; GILL, D.; ROMIG, C. Gaming science: the Gamification” of scientific thinking. Front. Psychol., v. 4, n. 607, 2013.

SALEN, K.; ZIMMERMAN, E. Rules of Play: Game Design Fundamentals. Cambridge: MIT Press, 2004, p. 4-8.

SANMARTí, N. Trabajo por proyectos: ¿Filosofía o metodología? Cuadernos de Pedagogía, v. 472, p. 44-46, 2016.

SANMARTÍ, N.; MÁRQUEZ, C. Aprendizaje de las ciencias basado en proyectos: del contexto a la acción. Ápice, revista de Educación Científica, v. 1, n. 1, p. 3-16, 2017.

SIMARRO, C.; COUSO, D. El papel del Tinkering en el aprendizaje formal e informal de las ciencias en primaria. Focusing on the learner. The DIYlab philosophy at school and Universitiy to Foster Student Agency and Collaborative Learning. Barcelona, Spain, 2016.

TORRES, C. M. El juego: una estrategia importante. Educere, la Revista Venezolana de Educación, v. 6, n. 19, p. 289-296, 2002. 
ULISACK, M. Games in Education: Serious Games. A Futurelab Literature Review. Londres: Futurelab, p. 1336, 2010.

WOOLFOLK, A. Psicología educativa. México, Pearson Educación, p. 374-415, 2006.

WERBACH, K.; HUNTER, D. For the Win: How Game Thinking Can Revolutionize Your Business. Philadelphia: Wharton Digital Press, p. 15-21, 2012.

YAGER, R. E. The constructivist learning model, towards real reform in science education. The Science Teacher, V. 58, n. 6, p. 52-57, 1991.

YVERN, A. ¿A qué jugamos? Buenos Aires: Bonum, p. 34-42, 1998.

Víctor López Simó: es licenciado en Física y doctor en didáctica de las ciencias experimentales. Es profesor de Ciencias en el Instituto de Secundaria Pau Vila (Sabadell, Barcelona), Profesor Asociado en el Departamento de Didáctica de la Matemática y las Ciencias Experimentales de la Facultad de Educación de la Universidad Autónoma de Barcelona y colaborador del CRECIM (Centro de Investigación para la Educación Científica y Matemática).

E-mail:victor.lopez@uab.cat

Jordi Domènech-Casal: es doctor en Biología y Licenciado en Humanidades. Es profesor de Ciencias en el Instituto de Secundaria Marta Estrada (Granollers, Barcelona) y Profesor Asociado en el Departamento de Didáctica de la Matemática y las Ciencias Experimentales de la Facultad de Educación de la Universidad Autónoma de Barcelona. Es asesor y embajador del programa STEMCat del Departament d'Ensenyament de la Generalitat de Catalunya.

E-mail: jdomen44@xtec.cat 